\title{
Structural Optimization of Adaptive Soft Fin Ray Fingers with Variable Stiffening Capability
}

\author{
Khaled Elgeneidy, Adel Fansa, Irfan Hussain, Khaled Goher
}

\begin{abstract}
Soft and adaptable grippers are desired for their ability to operate effectively in unstructured or dynamically changing environments, especially when interacting with delicate or deformable targets. However, utilizing soft bodies often comes at the expense of reduced carrying payload and limited performance in high-force applications. Hence, methods for achieving variable stiffness soft actuators are being investigated to broaden the applications of soft grippers. This paper investigates the structural optimization of adaptive soft fingers based on the Fin Ray ${ }^{\circledR}$ effect (Soft Fin Ray), featuring a passive stiffening mechanism that is enabled via layer jamming between deforming flexible ribs. A finite element model of the proposed Soft Fin Ray structure is developed and experimentally validated, with the aim of enhancing the layer jamming behavior for better grasping performance. The results showed that through structural optimization, initial contact forces before jamming can be minimized and final contact forces after jamming can be significantly enhanced, without downgrading the desired passive adaptation to objects. Thus, applications for Soft Fin Ray fingers can range from adaptive delicate grasping to high-force manipulation tasks.
\end{abstract}

Keywords-soft grippers, Fin Ray Effect, Optimization, Layer Jamming, Finite Element Analysis

\section{INTRODUCTION}

Soft and adaptable manipulators have the ability to operate efficiently in unknown or dynamically changing environments. Soft grippers are an example of morphological computation, where control complexity is greatly reduced by material softness and mechanical compliance [1]. Exploiting compliance and under-actuation in robotic hands enables improved adaptability to unstructured environments and the capability of manipulating delicate objects with varying shapes [2]. Interesting applications include harvesting and handling of crops and food products [3]. Rigid robots are composed of rigid links connected to actuators and, in such systems, the problems of structural behavior and control are decoupled. On the other hand, in soft robotics, these boundaries are blurred and there is a need to define new paradigms in engineering that challenge us to re-examine the relationships between the structural morphology and control [4]. The passive compliance and adaptability of soft robotic systems are often argued to offer unrivaled performance to handle deformable, fragile, and even time-varying objects [5]. Adaptive grasping is not only an attractive topic in the design of compliant and underactuated robotic hands [6], but also one of the main areas of application of soft robotics [7], [8]. Soft robotic fingers are often manufactured monolithically

K. E. and K. G. are with the Lincoln Centre for Autonomous Systems (LCAS), School of Engineering, University of Lincoln, Lincoln, UK. (corresponding author e-mail: kelgeneidy@lincoln.ac.uk). using highly flexible polymers. Pneumatically controlled fingers, such as the ones presented in [9], are a common application of soft robotics in the field of pick-and-place. These fingers are manufactured with inflatable pockets that allow the finger to conform to a variety of different shaped and sized objects. Another approach to introduce compliance in robotic fingers involves the use of hinges made of elastic materials, leading to simpler systems compared to rigid joints with mechanical springs [10]. Elastic hinges allow exploiting stored bending energy to return the actuated fingers to their initial position. In such devices, the grasping performance highly depends on the proper design of hinge based soft joints to avoid unwanted deformation in non-actuated directions while lifting relatively heavy objects [11].

An appealing solution to overcome the limitation of the above-mentioned fingers is the use of a passively adaptive structure referred to as the Fin Ray ${ }^{\circledR}$ Effect [12]. The Fin Ray structure is a biologically inspired phenomenon discovered by Leif Kniese in 1997 [13]. Leif Kniese and Rudolf Bannasch continued research into the anatomy of this biological phenomenon in under EvoLogics $\mathrm{GmbH}$ and developed the adaptive geometry named Fin Ray ${ }^{\circledR}$ [24]. The Fin Ray structure allows for a soft and compliant envelopment of objects without any embedded actuation. The fingers are simply pushed against the objects to be seized and they passively deform in reaction to the resulting contacts. The structure of the Fin Ray Effect (from now referred to as FRE) is composed of two vertical fins bound at one end, and horizontal ribs joining the fins throughout the length of the structure as illustrated in Fig. 1. The FRE enables the structure to conform to an external force, whilst maintaining rigidness out of its grasping plane. This has encouraged the utilization of the FRE structure as a highly adaptive gripper finger that is capable of grasping a variety of objects [13-17]. Despite being an excellent candidate for delicate grasping and soft manipulation applications due to their unique shapeadaptation, limited work has been directed towards optimizing their morphology for this application area. Previous work has been conducted on characterizing and improving the grasping performance of FRE inspired fingers with rigid ribs that do not significantly deform [16-18]. Our previous work has highlighted the benefits of an entirely flexible and soft FRE fingers for delicate grasping applications, with deforming ribs that can passively jam

A. F. is with the department of engineering, Ain Shams University, Cairo, Egypt.

I. H. is with the Autonomous and Robotics systems group, Khalifa University, Abu Dhabi. 
together to stiffen the finger and enhance force generation when needed [19].

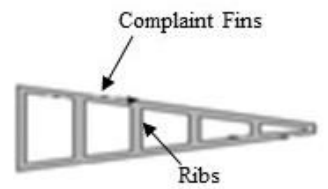

(a) Without Object

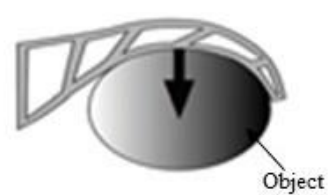

(b) With Object
Fig. 1: The composition of a typical Fin Ray finger consisting of two fins connected at one end which ribs joining the fins throughout the length of the finger. Both straight (a) and deformed (b) positions after contact are shown.

Stiffness variation is vital for soft-bodied structures to effectively interact with the environment and overcome their inherently limited force-generation. In literature, many approaches have been explored for active stiffening through direct activation of soft actuation technologies [20]. Among the suitable strategies for changing the stiffness of a soft robot, are the jamming-based systems [21] [22]. Towards the effort of optimizing the structure of FRE fingers, researchers have highlighted the benefit of tilting the ribs towards the grasped objects to create a preferred bending direction for better passive adaptation [17-19]. Additionally, recent work has investigated the optimization of FRE fingers that were coupled with electroadhesion capability [23]. Our previous work on Soft FRE fingers highlighted the potential for optimizing the passive stiffening ability based on layer jamming between flexible ribs to maximize both payload and adaptability. Using flexible materials, in general, reduces the rigidity of the finger and compromise its payload, which is a general challenge in soft robotics [20]. This challenge motivates our research to attempt to optimize Soft FRE fingers, in order to realize the difficult tradeoff between the desired passive shape-adaptation, and generating sufficient forces for high-force applications.

This paper extends previous work by optimizing the passive layer jamming behavior through finite element modeling of the proposed Soft FRE fingers with the objective of improving grasping performance in terms of (1) shape adaptation, and (2) grasping forces. Those are quantified by recording the tip displacement and measuring the force response for different structural variations to the Soft FRE morphology. The aim is to minimize initial contact forces (before jamming) when interacting with delicate targets, while having the ability to generate significantly higher forces (after jamming) when high-force tasks are required. In the same time maintaining the desired softness and flexibility of the FRE fingers for passive shape adaptation to targets.

The paper starts by introducing the design of the FRE structure and the unique passive layer jamming mechanism driving the stiffness variation of the Soft FRE fingers presented here. Details about the conducted finite element analysis (FEA) are then presented, outlining the adopted material model and the key design parameters investigated in this work. Furthermore, the FEA results are presented, starting with the effect of thickness variation on the structure's adaptability, followed by the effects of the initial rib angle and introducing angle increments between ribs, on simultaneously enhancing the passive adaptation and force response. Moreover, the experimental validation section explains how the optimized designs were 3D printed and experimentally tested to validate the FEA results. Lastly, the paper draws conclusions from the results and outlines plans for future work.

\section{StRUCTURAL OPTIMIZATION (FEA)}

\section{A. Passive Stiffening through Layer Jamming}

The proposed Soft FRE fingers exhibit a unique passive stiffening mechanism enabled by the combination of an entirely flexible Fin Ray structure, in addition to tilting the connecting ribs towards the contact surface [19]. When a Soft FRE finger is pressed against an object, the flexible and tilted ribs deform and start to make contact with each other, causing a layer jamming effect due to the friction between deforming ribs (Fig. 2). In this jammed state, the overall stiffness of the FRE structure increases due to layer jamming causing a significant increase in the gradient of the force response. Thus, the Soft FRE fingers benefit from minimal contact forces upon initial contact, which would be desired when adapting to delicate and sensitive targets, while significantly higher forces can also be achieved in the jammed state for high-force tasks or lifting of denser objects. In order to fully utilize the benefit of this passive layer jamming ability, the FRE structure needs to be optimized with the objective of maximizing the contact between flexible ribs in the jammed state. This is investigated in the next section by developing an accurate finite element model of the Soft FRE finger that effectively captures its deformation behavior, so that a range of structural variations can be simulated and evaluated in terms of improvement to grasping performance.

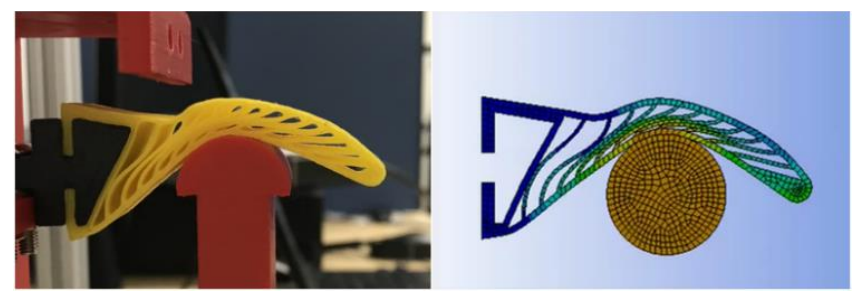

Fig. 2: Visualisation of a deformed Soft FRE finger after layer jamming comparing (a) Experimental test, (b) and corresponding FEA.

\section{B. Finite Element Analysis}

A static structural analysis for the deformation of the Soft FRE fingers was created using ANSYS software. As explained later in the paper, the material used in $3 \mathrm{D}$ printing the Soft FRE fingers, is called Ninjaflex (from Ninjatek), which is a highly flexible thermoplastic material with a Shore hardness of $85 \mathrm{~A}$. Previous work on characterizing the properties of 3D printed Ninjaflex has shown that the best performing hyperelastic model, was a second-order Ogden model [25]. The material coefficients based on this work were as follows: $\mu 1=0.133 \mathrm{MPa}, \alpha_{1}=3.05, \mu_{2}=-1214 \mathrm{MPa}, \alpha_{2}=$ -0.0054 , which results in a shear modulus $\mu$ of $3.58 \mathrm{MPa}$ and Young's modulus E of $12.42 \mathrm{MPa}$. A circular Object was simulated to translate a total of $15 \mathrm{~mm}$ against the simulated Soft FRE finger which was fixed at its base to simulate a rigid connection to the actuator as highlighted in Fig. 3. The 
simulation records the resulting tip displacement and reaction forces as the object presses against the finger and causes the flexible structure to deform and adapt.

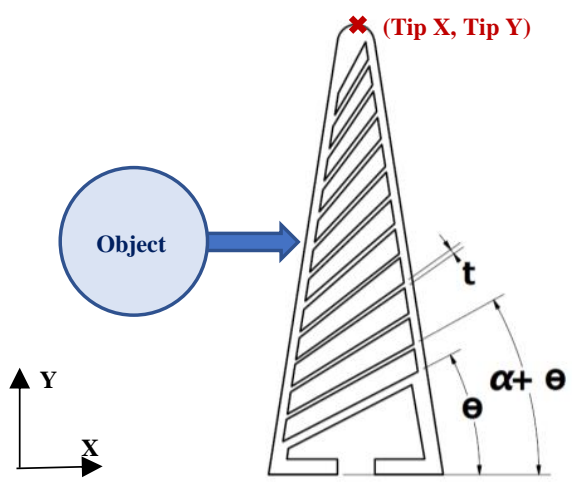

Fig. 3: Illustration for the FEA study showing the spherical object and the Soft Fin Ray structure labeled with key design parameters.

The Soft FRE design was parameterized on ANSYS to enable simulating the deformation of the fingers at a range of values for each tested parameter. Fig. 3 shows a cross-section through the Soft FRE design highlighting the key design parameters investigated in this work, which are: Thickness of ribs $(t)$, Initial angle of the first $\operatorname{rib}(\theta)$, and Angle increments between successive ribs $(\propto)$. The outer dimensions of the Soft FRE finger were held constant with an overall length of 61 $\mathrm{mm}$ and base width of $24 \mathrm{~mm}$, so that they are the same size as the original adaptive FRE fingers by Festo (DHAS-GF-60$\mathrm{U}-\mathrm{BU})$. Also, the number of ribs was fixed for a fair comparison between all simulated structure variations. A total of 12 ribs were chosen, which facilitated testing the thickness and angle variations at the required range without having to vary the number of ribs between different structure variations due to insufficient or excessive space at the tip.

Firstly, the effects of the thickness $(t)$ of the FRE structure on the tip deflection were evaluated to highlight the need for indirect approaches to enhance the force response without downgrading passive adaptation. Furthermore, the FEA investigated the effect of rib angle variations through the initial rib angle $(\theta)$ and introducing angle increments between successive ribs $(\propto)$. In other words, the last rib at the fingertip will be tilted further towards the grasped object in comparison to the first rib at the finger's base (Fig. 3). This is intended to enhance the layer jamming effect by increasing the number of ribs that come in contact during jamming. Improving the contact between ribs will consequently cause increased stiffening after jamming, and hence increasing maximum generated contact forces.

\section{FEA RESULTS}

\section{A. Thickness variation}

The first evaluated parameter was the thickness of the Soft FRE structure, which corresponds to the overall stiffness of the fingers. The thickness was varied from $0.6 \mathrm{~mm}$ to $1.8 \mathrm{~mm}$ in increments of $0.6 \mathrm{~mm}$, which corresponds to the nozzle diameter used later to $3 \mathrm{D}$ print the Soft FRE fingers.
Maintaining dimensions to be integers of the nozzle diameter helps to ensure a clean print with flexible filaments. Fig. 4 shows the recorded $\mathrm{X}$ and $\mathrm{Y}$ tip displacement of each of the tested thickness values against the $15 \mathrm{~mm}$ displacement of the object pushing the Soft FRE fingers. The object is displacing along the positive $\mathrm{x}$-axis direction only. It can be seen that the tip of the $1.8 \mathrm{~mm}$ structure traveled $9.8 \mathrm{~mm}$ along the $\mathrm{x}$-axis at the end of the $15 \mathrm{~mm}$ displacement imposed by the object, compared to only $3.4 \mathrm{~mm}$ tip displacement by the $0.6 \mathrm{~mm}$ thick structure. Less tip travel in the $\mathrm{x}$-axis means better adaptation since the object displacement is being absorbed by the FRE structure. The line corresponding to no displacement is shown in Fig. 4. Similarly, the 1.8 thick structure moved only $5 \mathrm{~mm}$ towards the object along the y-axis, compared to $8.5 \mathrm{~mm}$ displacement by the $0.6 \mathrm{~mm}$ thick structure. In this case, more travel along the $y$-axis is desired as it means that the tip is closing on the object pushing the finger along the $\mathrm{x}$ axis. Fig. 4 also includes the tip displacement for the FRE structure with a combination of $0.6 \mathrm{~mm}$ rib thickness and 1.2 $\mathrm{mm}$ outer fins thickness. It can be observed that increasing the outer thickness of the finger did not make a significant impact on the tip displacement as, yet it improves the structural integrity of the $3 \mathrm{D}$ printed samples.

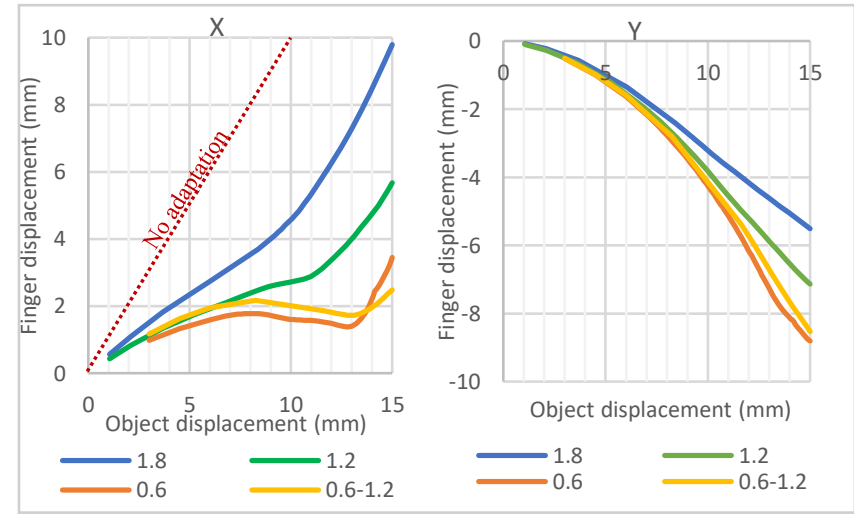

Fig. 4: Effects of varying the thickness of the Fin Ray structure on the tip displacement along the $\mathrm{X}$ and $\mathrm{Y}$-axis.

Although thickening the FRE structure can increase contact forces, it is not desired due to the consequent reduction in the desired passive adaptation along both axes. Hence, alternative structural modifications are desired that could indirectly enhance force generation while keeping the rib thickness at $0.6 \mathrm{~mm}$ to maintain the finger's softness and flexibility.

\section{B. Force Response}

An alternative approach to enhance force generation from FRE fingers while maintaining their desired passive adaptation is through tuning of the rib angles to optimize the witnessed layer jamming behavior. Previous work has shown that tilting the ribs towards the grasped object creates a preferred bending direction that generally improves grasping performance [17-19] [23]. Hence, only positive angles have been investigated in this work. Fig. 5 shows the force response from four Soft FRE fingers with a fixed rib thickness of $0.6 \mathrm{~mm}$ and initial rib angle $\theta=10^{\circ}$, but each follows different angle increments from zero (all ribs are parallel) to $\propto=3^{\circ}$. It is observed that by introducing angle increments 
between ribs, the slope of the force response after layer jamming can be increased, while the initial contact forces remain the same. At $\propto=3^{\circ}$ the final resultant force at the end of the $15 \mathrm{~mm}$ displacement is $39.4 \%$ higher than the same finger with no angle increments between ribs.

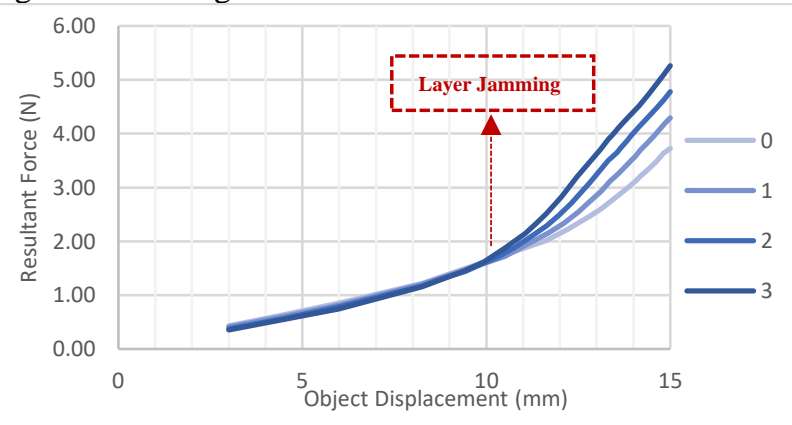

Fig. 5: Resultant force response at varying angle increments between ribs and an initial rib angle of $10^{\circ}$

Furthermore, Fig. 6 illustrates the effects of varying both $\theta$ and $\propto$ on the initial (at $3 \mathrm{~mm}$ displacement) and final (at 15 $\mathrm{mm}$ displacement) resultant contact forces. It can be seen that $\theta$ has no significant effect on the final force value but influences the initial contact force. Increasing the angle of the first rib from $10^{\circ}$ to $25^{\circ}$ helps in minimizing contact forces upon initial contact, which would be desired when interacting with delicate targets. As previously shown, increasing the angle increments between ribs can significantly enhance maximum contact forces, but also has the added benefit of further reducing the initial contact forces. Hence, a Soft FRE finger with $\theta=25^{\circ}$ and $\propto=3{ }^{\circ}$ will have significantly enhanced force generation after layer jamming, while at the same time minimizing initial contact forces before layer jamming.

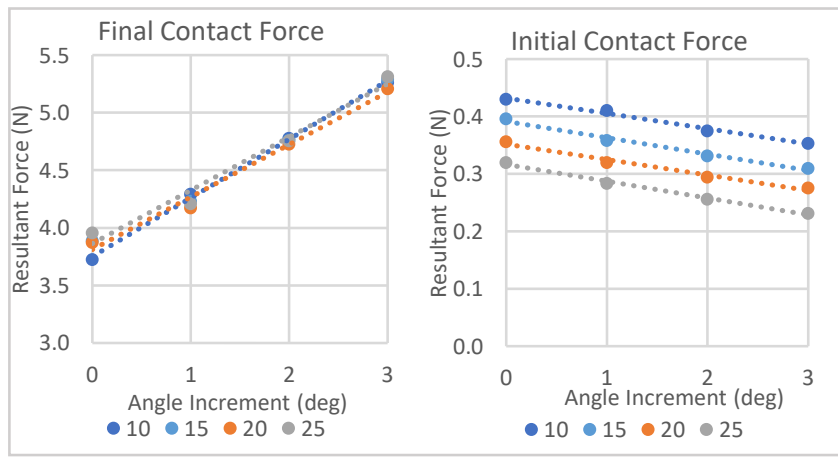

Fig. 6: Effect of initial rib angle and angle increments on the initial and final resultant contact forces

\section{Passive Adaptation}

Fig. 7 shows the tip displacement along the $\mathrm{X}$-axis for soft fingers with angle increments $(\propto)$ varying from $0^{\circ}$ to $3^{\circ}$ at 1 ${ }^{o}$ increments. It is generally observed that upon initial contact with the object, the fingertips are pushed along the same direction of object displacement, yet with a lesser value as the FRE structure deforms and adapts to the external object. However, Soft FRE fingers with larger angle increments are able to better adapt to the external object, moving even behind the initial tip position (negative displacement values) when $\propto$ $>1^{\circ}$. At $12 \mathrm{~mm}$ of object displacement against the finger, the Soft FRE fingertip with $\propto=3^{\circ}$, has moved nearly $4 \mathrm{~mm}$ further towards the object compared to the one with no angle increments $\left(\propto=0^{\circ}\right)$. Furthermore, after layer jamming (dotted line on Fig. 7), all fingers are unable to further adapt to the object since the structure has stiffened and hence, the whole finger is displaced in the direction of object displacement. This explains the rise in the slope at the end of tip displacement response beyond the layer jamming point.

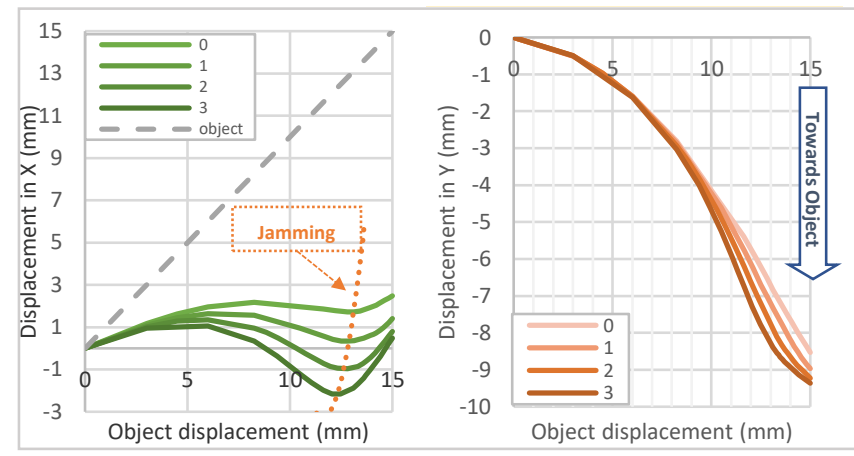

Fig. 7: Tip displacement response along the $\mathrm{X}$ and $\mathrm{Y}$ axis at variable angle increments and a fixed initial angle of $10^{\circ}$.

Looking at the tip displacement along the Y-axis, Fig. 7 shows how increasing the angle increments between ribs also improves shape adaptation along the $\mathrm{Y}$-axis although less than the witnessed adaptation along $X$-axis. At $13 \mathrm{~mm}$ object displacement, the Soft FRE fingertip with $\propto=3^{\circ}$, has moved nearly $2 \mathrm{~mm}$ further towards the object compared to the one with no angle increments $\left(\propto=0^{\circ}\right)$. Thus, overall, increasing angle increments benefit the shape adaptation of Soft FRE fingers as the fingertip can close further on a target object. Fig. 8 illustrates the effects of varying both $\theta$ and $\propto$ on the maximum adaptation along $\mathrm{X}$ and $\mathrm{Y}$ axis, which is quantified as the difference between the object's displacement and the fingertip position. Larger values mean better adaptation, with $15 \mathrm{~mm}$ in $\mathrm{x}$-axis corresponding to the fingertip remaining in its initial position despite the $15 \mathrm{~mm}$ displacement of the object. It is observed, that by combining smaller starting angles and larger angle increments, the best overall adaptation can be achieved. Thus, although tilting ribs creates a preferred bending direction for FRE structures, excessively tilting the initial angle has an adverse effect on adaptation for Soft FRE fingers.

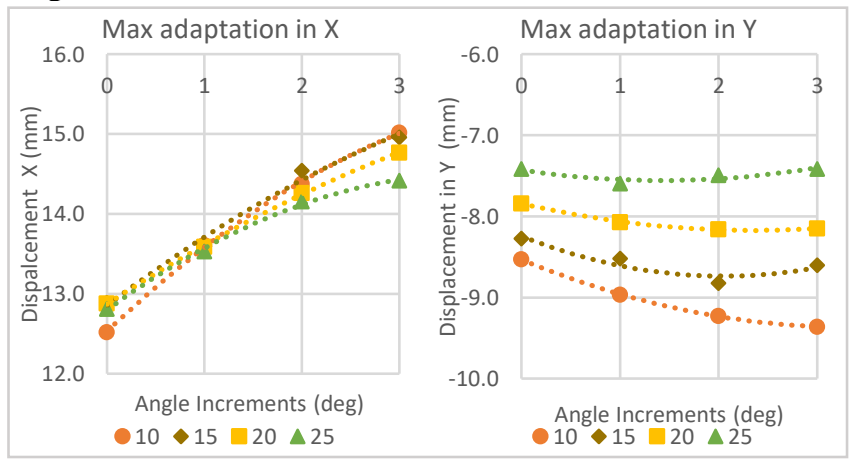

Fig. 8: Effect of initial rib angle and angle increments on the tip adaptation for a $15 \mathrm{~mm}$ object displacement against the soft fin ray fingers 


\section{EXPERIMENTAL VALIDATION}

\section{A. $3 D$ printing}

The soft FRE fingers can be 3D printed directly from the flexible Ninjaflex material using standard FDM printers (such as the Lulzbot TAZ 6 printer used here). This allows fast and simple customization of the fingers based on application needs. The print parameters are detailed in our previous work [19]. In order to ensure good surface finish of the printed Soft FRE fingers, it is recommended to choose the dimensions of the geometry so that they are integer values of nozzle diameter to minimize idle nozzle travel. This reduces material stringing during idle travels, which flexible filaments are prone to. In other words, the printer nozzle will be continuously printing with minimal retractions and crossing over printed areas. This can be achieved by generating the morphology of the Soft FRE entirely in Cura slicing software as previously explained [19]. In this case, the CAD model is a solid prism with the required overall dimensions, while the ribs will be generated according to the chosen infill settings, such as line width, infill density, and infill angle. However, default infill settings do not include support further customization such as to create the variable infill angles introduced here. Hence, the FRE structure was generated using CAD software (Inventor) and imported to Cura for slicing as shown in Fig. 9. It can be observed that there are minimal idle travels (dark blue lines), which contributes to better print quality.

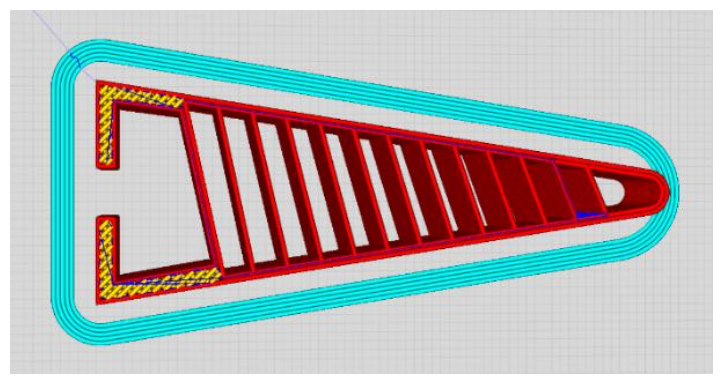

Fig. 9: Model of the Soft Fin Ray fingers sliced using Cura

\section{B. Experimental Setup}

In order to validate the FEA results a test rig (shown in Fig. 10) was constructed to record the force response generated when testing 3D printed samples of optimized Soft FRE fingers. A round target object was 3D Printed from PLA with the same diameter used in the simulation $(20 \mathrm{~mm})$. The object was mounted on a force/torque sensor (Schunk Mini40) calibrated for the range 0 to $20 \mathrm{~N}$ and attached to the frame setup. A motor controlled (Nema 17) vertical linear stage displaces the Soft FRE against the test object by a $15 \mathrm{~mm}$ stroke in $1 \mathrm{~mm}$ steps. At each step, the generated forces from the force/torque sensor are logged and compared to the corresponding data from FEA. The tested FRE finger is fixed on the vertical stage using a $3 \mathrm{D}$ printed mount. Three samples for the best performing FRE structures from the FEA optimization were 3D printed and experimentally tested. Each sample was tested three times to evaluate the consistency of the results between different samples and across repetitions.

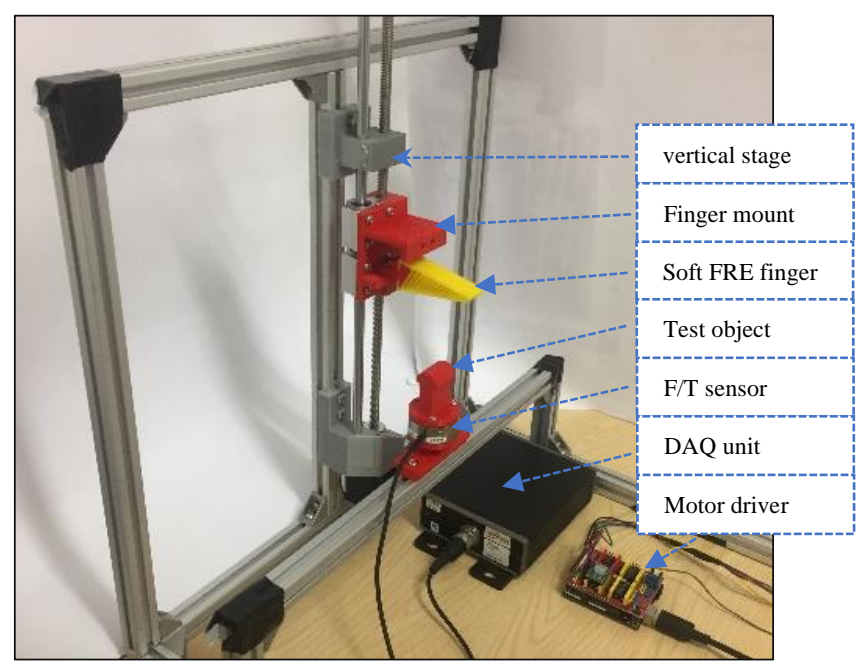

Fig. 10: Labelled experimental setup for testing Soft FRE fingers

\section{Experimental Validation results}

Fig. 11 compares the force response from FEA and mean experimental data for the two best performing Soft FRE fingers $\left(\alpha=3\right.$ and $\theta=25^{\circ}$, and $\propto=3$ and $\left.\theta=10^{\circ}\right)$. It is clear that the FEA results closely matched the experimental data from physical tests, which confirms the accuracy of the chosen hyperelastic model within the operating strain range. The experimental results were found to be highly repeatable given the same print settings are and part orientation are used. Standard deviation in the final resultant force between samples for the same design was $0.22 \mathrm{~N}$, while standard deviation between repetitions was only $0.082 \mathrm{~N}$. Furthermore, Fig. 2 visually compares the deformation of a Soft FRE finger sample to highlight how the FEA effectively captured the deformation behavior including the layer jamming between the flexible ribs.

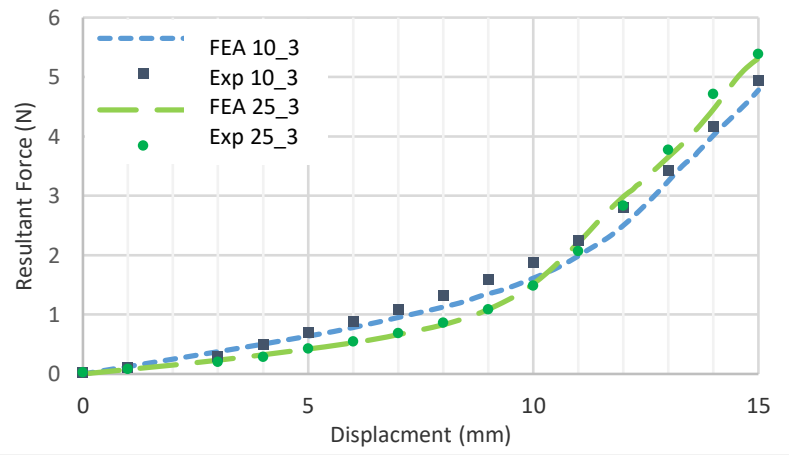

Fig. 11: Resultant force response (FEA vs. experimental) for best performing structure (initial angles of $10^{\circ}$ and $25^{\circ}$ with angle increments of $3^{\circ}$ )

\section{CONCLUSIONS}

In this paper, we have presented a customizable and 3D printable adaptive soft fingers based on the Fin Ray® effect (Soft FRE), which exhibit a passive stiffening mechanism facilitated by the layer jamming between flexible and tilted connecting ribs. This broadens the applications of adaptive fingers based on the FRE to include not only delicate grasping, but also high-force applications that are currently challenging for typical soft robotic fingers. We showed that through structural optimization of the Soft FRE's layer jamming 
between deforming ribs, initial contact forces (before jamming) were reduced and final contact forces (after jamming) were significantly increased, while maintaining and even slightly improving the desired passive adaptation. By introducing angle increments $(\propto)$ between successive flexible ribs, the optimized Soft FRE structure showed a reduction in initial contact forces, while at the same time increasing the maximum contact forces by up to $40 \%$ after stiffening. In addition to improving maximum tip displacement towards the grasped object by nearly $4 \mathrm{~mm}$ along the $\mathrm{x}$-axis and $2 \mathrm{~mm}$ along $\mathrm{Y}$-axis, compared to the same finger with no angle increments. Furthermore, the starting rib angle $(\theta)$ showed a less significant effect on Soft FRE performance. More specifically, although tilting the ribs generally towards the contact surface is desired, decreasing the initial rib angle reduces the initial contact forces and aids improvement in passive adaptation along the y-axis. Finally, the FEA results were validated experimentally by $3 \mathrm{D}$ printing and testing the best performing optimized Soft FRE fingers. The force response recorded from the experiment well-matched the simulation results across different samples and repetitions.

\section{FUTURE WORK}

The next stage of this work will investigate further structural and topological optimizations that can enhance the grasping performance of grippers utilizing the developed Soft FRE fingers, particularity in regard to reducing slippage. The grasping performance of the optimized Soft FRE fingers will be evaluated through grasping tests across a diverse set of objects that ranges from delicate to rigid. Additionally, it would be useful to actively control switching between jammed and relaxed states through embedded or external sensory feedback. This would enable controlling the generated contact forces during grasping, depending on the displacement against the target object and the number of jammed layers.

\section{ACKNOWLEDGEMENTS}

This research was funded by the University of Lincoln's school of engineering. The authors would like to thank Dr Jonathan Griffiths for his support with Ansys.

\section{REFERENCES}

[1] Dollar, Aaron M., and Robert D. Howe. "The highly adaptive SDM hand: Design and performance evaluation." The international journal of robotics research 29.5 (2010): 585-597.

[2] I. Hussain et al., "Modeling and Prototyping of an Underactuated Gripper Exploiting Joint Compliance and Modularity," in IEEE Robotics and Automation Letters, vol. 3, no. 4, pp. 2854-2861, Oct. 2018. doi: 10.1109/LRA.2018.2845906.

[3] Wang, Z., Torigoe, Y., and Hirai, S. (2017). A prestressed soft gripper: Design, modeling, fabrication, and tests for food handling. IEEE Robotics and Automation Letters, 2(4):1909-1916.

[4] Majidi, Carmel. "Soft robotics: a perspective-current trends and prospects for the future." Soft Robotics 1.1 (2014): 5-11.

[5] Z. Wang, M. Z. Q. Chen, and J. Yi, .Soft robotics for engineers,. HKIE. Transactions, vol. 22, no. 2, pp. 88.97, 2015.

[6] L. Birglen, T. Lalibert'e, and C. Gosselin, Underactuated Robotic Hands. Springer Berlin Heidelberg, 2008.

[7] J. Hughes, U. Culha, F. Giardina, F. Guenther, A. Rosendo, and F. Iida, Soft manipulators and grippers: A review,. Frontiers in Robotics and AI, vol. 3, p. 69, 2016.
[8] F. Iida and C. Laschi, .Soft robotics: Challenges and perspectives Procedia Computer Science, vol. 7, pp. 99 . 102, 2011, proceedings of the 2nd European Future Technologies Conference and Exhibition 2011 (FET 11).

[9] Manti, Mariangela, et al. "A bioinspired soft robotic gripper for adaptable and effective grasping." Soft Robotics 2.3 (2015): 107116.

[10] Hussain, Irfan, et al. "On the role of stiffness design for fingertip trajectories of underactuated modular soft hands." 2017 IEEE International Conference on Robotics and Automation (ICRA). IEEE, 2017.

[11] Salvietti, Gionata, et al. "Design of the passive joints of underactuated modular soft hands for fingertip trajectory tracking." IEEE Robotics and Automation Letters 2.4 (2017): 2008-2015.

[12] FESTO, "Multichoicegripper [online]," https://www.festo.com/net/ SupportPortal/Files/333986/Festo MultiChoiceGripper en.pdf, 2014.

[13] Pfaff O, Simeonov S, Cirovic I, and Stano P. Application of Finray effect approach for production process automation. Annals of DAAAM \& Proceedings, 2011, 1247-1248.

[14] Hussain, Irfan, et al. "Design and Prototype of Supernumerary Robotic Finger (SRF) Inspired by Fin Ray® Effect for Patients Suffering from Sensorimotor Hand Impairment." 2019 2nd IEEE International Conference on Soft Robotics (RoboSoft). IEEE, 2019.

[15] Anwar, Muddasar, et al. "Modeling and prototyping of a soft closed-chain modular gripper." Industrial Robot: the international journal of robotics research and application 46.1 (2019): 135-145.

[16] C. I. Basson, G. Bright, A. J. Walker, and A. J. Walker, "Testing Flexible Grippers for Geometric and Surface Grasping Conformity in Reconfigurable Assembly Systems," South African J. Ind. Eng., vol. 29, no. May, pp. 128-142, May 2018.

[17] Crooks W, Vukasin G, O'Sullivan M, Messner W, and Rogers C. Fin Ray Effect Inspired Soft Robotic Gripper: From the RoboSoft Grand Challenge Toward Optimization. Frontiers in Robotics and AI, 2016, 3.

[18] W. Crooks, S. Rozen-Levy, B. Trimmer, C. Rogers, and W. Messner, "Passive gripper inspired by Manduca Sexta and the fin ray ${ }^{\circledR}$ effect," Int. J. Adv. Robot. Syst., vol. 14, no. 4, pp. 1-7, 2017.

[19] K. Elgeneidy, P. Lightbody, S. Pearson, and G. Neumann, "Characterising 3D-printed soft fin ray robotic fingers with layer jamming capability for delicate grasping," RoboSoft 2019 - 2019 IEEE Int. Conf. Soft Robot., pp. 143-148, 2019.

[20] M. Manti, V. Cacucciolo, and M. Cianchetti, "Stiffening in Soft Robotics: A Review of the State of the Art," IEEE Robot. Autom. Mag., vol. 23, no. 3, pp. 93-106, 2016.

[21] V. Wall, R. Deimel, and O. Brock, "Selective stiffening of soft actuators based on jamming," IEEE Int. Conf. Robot. Autom., pp. 252-257, 2015.

[22] M. Langer, E. Amanov, and J. Burgner-Kahrs, "Stiffening Sheaths for Continuum Robots," Soft Robot., vol. 5, no. 3, p. soro.2017.0060, 2018.

[23] R. Chen et al., "Bio-Inspired Shape-Adaptive Soft Robotic Grippers Augmented with Electroadhesion Functionality," Soft Robot., no. August, 2019.

[24] S. Alben, P. G. Madden, and G. V. Lauder, "The mechanics of active fin-shape control in ray-finned fishes," Journal of The Royal Society Interface, vol. 4, no. 13, pp. 243-256, 2007.

[25] T. Reppel and K. Weinberg, "Experimental determination of elastic and rupture properties of printed Ninjaflex," Tech. Mech., vol. 38, no. 1, pp. 104-112, 2018. 